\title{
Sex chromosome turnovers and genetic drift: a simulation study
}

\author{
PAUL A. SAUNDERS* (D), SAMUEL NEUENSCHWANDER* $\uparrow \& N I C O L A S$ PERRIN* \\ *Department of Ecology and Evolution, Université de Lausanne, Lausanne, Switzerland \\ $\dagger$ Vital-IT, Swiss Institute of Bioinformatics, Lausanne, Switzerland
}

Keywords:

genetic drift;

individual-based simulations;

quantiNemo;

sex determination;

sex ratio selection;

transitions.

\begin{abstract}
The recent advances of new genomic technologies have enabled the identification and characterization of sex chromosomes in an increasing number of nonmodel species, revealing that many plants and animals undergo frequent sex chromosome turnovers. What evolutionary forces drive these turnovers remains poorly understood, but it was recently proposed that drift might play a more important role than generally assumed. We analysed the dynamics of different types of turnovers using individual-based simulations and show that when mediated by genetic drift, turnovers are usually easier to achieve than substitutions at neutral markers, but that their dynamics and relative likelihoods vary with the type of the resident and emergent sex chromosome system (XY and/or $\mathrm{ZW})$ and the dominance relationships among the sex-determining factors. Focusing on turnovers driven by epistatically dominant mutations, we find that drift-mediated turnovers that preserve the heterogamety pattern are $2-4 \times$ more likely than those along which the heterogametic sex changes. This ratio nevertheless decreases along with effective population size and can even reverse in case of extreme polygyny. This can be attributed to a 'drift-induced' selective force, known to influence transitions between male and female heterogamety, but which according to our study does not affect turnovers that preserve the heterogametic sex.
\end{abstract}

\section{Introduction}

Genetic sex determination is widespread across eukaryotes (Bachtrog et al., 2014; Beukeboom \& Perrin, 2014), with male heterogamety (XX females, $\mathrm{XY}$ males) and female heterogamety (ZW females, ZZ males) being the most common systems in plants and animals. Thanks to recent advances in genomic technologies, sex chromosome systems are now characterized across a wide range of nonmodel organisms, revealing that many clades experience frequent sex chromosome turnovers, whereby an 'emergent' mutant sex determiner replaces the 'resident' sex determiner at the top of the sexdetermining cascade. This is the case for instance in fishes (Mank \& Avise, 2009), reptiles (Ezaz et al., 2009), amphibians (Miura, 2008; Dufresnes et al., 2014; Furman \& Evans, 2016), Diptera (Vicoso \& Bachtrog,

Correspondence: Paul A. Saunders, Department of Ecology and Evolution, Université de Lausanne, CH-1015 Lausanne, Switzerland.

Tel.: +41 21692 5615; fax: +41 21692 4165;

e-mail: paul.alan.saunders@gmail.com
2015), isopods (Becking et al., 2017) and Salicaceae (Muyle et al., 2018).

What forces drive sex chromosome turnovers has been the focus of discussions for over four decades. Several selective forces have been proposed as potential drivers (reviewed in van Doorn, 2014a; van Doorn, $2014 b$ ), but all have received very little empirical support so far (see, e.g. Roberts et al., 2009; Cordaux et al., 2011). In parallel, it was also proposed that turnovers could occur in the absence of any selective advantage for the emergent sex determiner, that is a turnover can be mediated solely by genetic drift (Bull \& Charnov, 1977). In the time since its inception, this neutral model of sex chromosome turnover had been largely neglected, at best considered to be 'relevant mainly as a null model or first approximation' (van Doorn, 2014b). However, a recent theoretical study suggests that neutral sex chromosome turnovers might be more frequent than generally assumed (especially if effective population size is small), due to an emergent 'drift-induced' selective force that accelerates the spread of epistatically 
dominant mutant sex determiners (Veller et al., 2017). This study focused on transitions between male and female heterogamety (XY to ZW and ZW to XY turnovers), yet in several taxa known to experience high rates of turnovers, the heterogametic sex seems to be conserved across species or clades. In salmonids, sex chromosomes can be found on at least six different linkage groups, but all species are XY (Phillips, 2013). In Lacertidae and Varanidae lizards, which also underwent changes in sex chromosomes, all species are ZW (Ezaz et al., 2009; Pokorná \& Kratochvíl, 2009). Sex chromosome turnovers have also occurred multiple times in Ranid frogs, but only one has resulted in a change in the heterogametic sex (Sumida \& Nishioka, 2000; Miura, 2008). Furthermore, the rate of turnovers that preserve the heterogamety pattern (XY to $\mathrm{XY}$ and $\mathrm{ZW}$ to $\mathrm{ZW}$ turnovers) is likely underappreciated in many taxa because of technical limitations (Brelsford et al., 2017). Some of the currently used methods to detect sex determination rely solely on the identification of sex-linked markers (which allow to distinguish between male and female heterogamety) but does not single out the sex chromosome pair, preventing the identification of turnovers within groups homogeneous for heterogamety (Gamble et al., 2015; Lambert et al., 2016). For now, it remains unknown whether sex chromosome turnovers that preserve the heterogamety pattern and turnovers that change the heterogametic sex are equally likely to occur under a selectively neutral regime. To fill this knowledge gap, we use a simulation-based approach to compare the neutral dynamics of the two types of turnovers and address several questions: assuming the same rate of emergence of male and female determiners, do the two types of turnover have the same likelihood of occurring under the neutral model of sex chromosome turnover? How is their relative likelihood affected by demographic and life-history variables known to affect effective population size (carrying capacity and mating system)? Are turnovers that conserve heterogamety affected in the same way as turnovers that change heterogamety by the 'driftinduced' selection described in Veller et al. (2017)?

\section{Materials and methods}

\section{Genetic model}

We restricted our study to nonhomologous turnovers, which involve two chromosomal pairs. A sex chromosome pair carries the 'resident' sex-determining locus with two alleles: either $X$ and $Y$ (male heterogamety), or $Z$ and $W$ (female heterogamety). An autosomal pair carries a gene involved in the sex-determining cascade, originally fixed for an allele $a$, which can mutate to become a 'novel' sex determiner, called $M$ or $F$ depending on whether it has a masculinizing or feminizing effect. We focus predominantly on turnovers that involve epistatically dominant mutant sex determiners (mutants with so-called strong sex-determining effects sensu; Bull \& Charnov, 1977). These turnovers are the most commonly studied in theoretical studies (e.g. van Doorn $\delta$ Kirkpatrick, 2007, 2010; Vuilleumier et al., 2007; Kozielska et al., 2010; Blaser et al., 2012), but note that mutant sex determiners are not necessarily epistatically dominant, and sex determiners could have 'mild' or 'weak' sex-determining effects (Bull \& Charnov, 1977).

Sex chromosome turnovers are of two different types: they either conserve the heterogamety pattern (thereafter called 'cis-heterogamety turnovers', e.g. when a epistatically dominant $M$ allele invades an XY system or a dominant $F$ invades a ZW system) or induce a switch in the heterogametic sex ('trans-heterogamety' turnovers, e.g. when a dominant $F$ invades an XY system, or a dominant $M$ invades a ZW system). Throughout a turnover, the two loci are polymorphic, resulting in a polygenic phase, with more than two sexual genotypes. For example, if a dominant $M$ allele emerges in an XY system, males have either an $X Y$ aa or $X X$ aM genotype, whereas all females are $X X a a$; and if a dominant $F$ allele emerges in an $X Y$ system, males have either an $X Y$ a a or $Y Y$ a a genotype, and females have one of the following four genotypes: $X X a a, X X a F, X Y a F$ or $Y Y$ $a F$. A turnover is achieved once polymorphism at the ancestral sex-determining locus is lost.

\section{Simulations}

Individual-based simulations were performed with a modified version of quantiNemo 1.0.3 (Neuenschwander et al., 2008). The starting point was a finite population with a simple single-locus sex determination system and an equal number of males and females (either $X Y a a$ and $X X a a$ or $Z Z a a$ and $Z W a a$ ). At each generation (nonoverlapping), gametes were drawn randomly from one father and one mother (with replacement) and paired to constitute individuals of the next generation. The autosomal allele $a$ was allowed to mutate to its sex-determining form (either $M$ or $F$ depending on simulations) at a rate $\mu=10^{-4}$ (intentionally high, as our main goal is to compare different turnover rates, not to have a plausible estimation of absolute turnover time), the reverse mutations were not allowed (one-way mutation model). No mutations occurred at the resident sex-determining locus.

A first set of simulations aimed at comparing the dynamics of the two types of neutral turnovers (cisand trans-heterogamety turnovers) assuming epistatically dominant sex-determining mutations (cases 2A and 3A in Bull \& Charnov, 1977), with a default population size $N=1000$. A second set aimed at assessing the effect of population size on these neutral turnovers. Simulations were run again with $N$ ranging from 50 to 10000 . A third set aimed at assessing the effect of mating system on turnovers, with random mating replaced 
by polygyny: a number $n b$ of breeding males (ranging from 10 to 400 ) were selected randomly to breed at each generation, and for each offspring, a mother and one of these breeding males were assigned with equal probability. Finally, to take a glance at the impact of drift on the spread of nondominant sex determiners, we performed an extra set of simulations limited to a smaller parameter space (initially XY system, random mating, $N=\{100,1000$ or 5000 $\}$ ), in which the mutant sex-determining allele is nondominant. We analysed the spread of a mildly male-determining mutant (case 2B in Bull \& Charnov, 1977), mildly female-determining mutant (case 3Bl) and weakly female-determining mutants (cases 2C1 and 2C2).

All simulations were run until a turnover had occurred, and time to turnover (number of generations until the end of the simulation) was systematically assessed for 1000 replicates for each set of parameters. To facilitate comparisons with predictions of the neutral theory of molecular evolution, we ran additional simulations where the mutant allele at the autosomal locus is a simple neutral marker, not involved in sex determination. The expected mean time for fixation of a neutral allele assuming one-way mutations is proportional to effective population size $\mathrm{Ne}$ and is given by $\bar{t}=\sum_{j=1}^{\infty} \frac{4 N_{e}}{j(j-1+\theta)} \quad$ with $\theta=4 N_{e} \mu \quad$ (Ewens, 2012). The mean time to fixation calculated from our simulations (averaged over 1000 replicates for each set of parameters) precisely followed this expected analytical value (Fig. S1), validating our approach.

\section{Results and discussion}

With population size $N=1000$ and random mating, the two types of sex chromosomes turnovers involving epistatically dominant mutant sex determiners occurred in average faster than substitutions at the neutral autosomal locus not involved in sex determination (Fig. 1). This is in line with results by Veller et al. (2017) where mutations causing trans-heterogamety turnovers fixed at a higher rate than other neutral mutations. In addition, we found that cis-heterogamety turnovers ( $\mathrm{XY}$ to $\mathrm{XY}$ and $\mathrm{ZW}$ to $\mathrm{ZW}$ ) occurred in average $2.5 \times$ faster than trans-heterogamety turnovers. To test whether this translates into a higher probability for turnovers conserving heterogamety to occur, we slightly modified our model: starting with an XY system, we allowed both mutant male and female determiners to emerge in the same simulation (at the same rate but at different loci, with $F$ dominant over $M$ ). Over 1000 replicates, a $\mathrm{XY}$ to $\mathrm{XY}$ turnover happened in 735 simulations and a $\mathrm{XY}$ to $\mathrm{ZW}$ turnover in 265 simulations $(2.77 \times)$. Relative time to turnover is therefore a reliable indicator of relative turnover probability: therefore, under these conditions, turnovers are two to three times more likely to conserve heterogamety. This difference probably stems from the fact that cis- and trans-heterogamety turnovers are inherently asymmetric. First, the number of sexual genotypes produced throughout a turnover differs (see Methods). Second, because of selection for a balanced sex ratio during the polygenic phase, the sex chromosome of the ancestral pair that goes to fixation once the transition is over is different: $\mathrm{X}$ (or $\mathrm{Z}$ ) in a cisheterogamety turnover, $\mathrm{Y}$ (or $\mathrm{W}$ ) in a trans-heterogamety turnover. Consequently, the continuous paths of neutral equilibria linking one sex determination system to the other (along which genotypes are maintained at such frequencies that the population sex ratio is balanced; Bull \& Charnov, 1977) differ between the two types of transitions (Fig. 2). For cis-heterogamety turnovers (where $\mathrm{X}$ or $\mathrm{Z}$ goes to fixation), this path is a straight diagonal line between the two sex determination systems, whereas for trans-heterogamety turnovers (where Y or W goes to fixation), it is curved, due to the more complex interplay of the different male and female genotypes. In addition, the length of these paths differ: it is shorter for cis-heterogamety transitions, as the $\mathrm{Y}$ (or W) chromosome frequency goes from 0.25 to 0.0 (arc-length: 0.354), whereas in trans-heterogamety transitions, it has to go from frequency 0.25 to 1.0 (arclength: 0.695).

Population size had drastically different impacts on the two types of turnovers (Fig. 3a, S2). Cis-heterogamety turnovers are affected in the same way as neutral mutations not involved in sex determination, whereas trans-heterogamety turnovers become relatively faster (and thus more likely) as population size decreases (Fig. 3a). The latter can be attributed to the recently described 'drift-induced' selective force that constrains neutral trans-heterogamety turnovers (Veller et al., 2017). This force is triggered by the stochastic fluctuations around the path of neutral equilibria and drags the system back to the path, with a bias towards the dominant sex determiner. As already noted by van Doorn (2014a), this force stems from selection for a balanced sex ratio. Indeed, fluctuations around the line of equilibria cause sex ratio to deviate from a $1: 1$ ratio. These fluctuations are much greater in smaller populations (Fig. S3), resulting in greater sex ratio biases along turnovers (Fig. S4) and therefore stronger selection for a balanced sex ratio. We propose that the response to sex ratio selection is magnified in trans-heterogamety turnovers because different types of crosses produce different sex ratios (e.g. $\mathrm{XY}$ aa $\times \mathrm{XX}$ aF crosses produce three females for one male, whereas YY aa $\times \mathrm{XX}$ aa produce only males), which provides the raw material on which selection can operate. In contrast, all possible crosses in cis-heterogamety turnovers produce balanced sex ratios, limiting the response to sex ratio selection. Thus, the 'drift-induced' force described in Veller et al. (2017) does not operate in cis-heterogamety turnovers triggered by epistatically dominant mutations. 


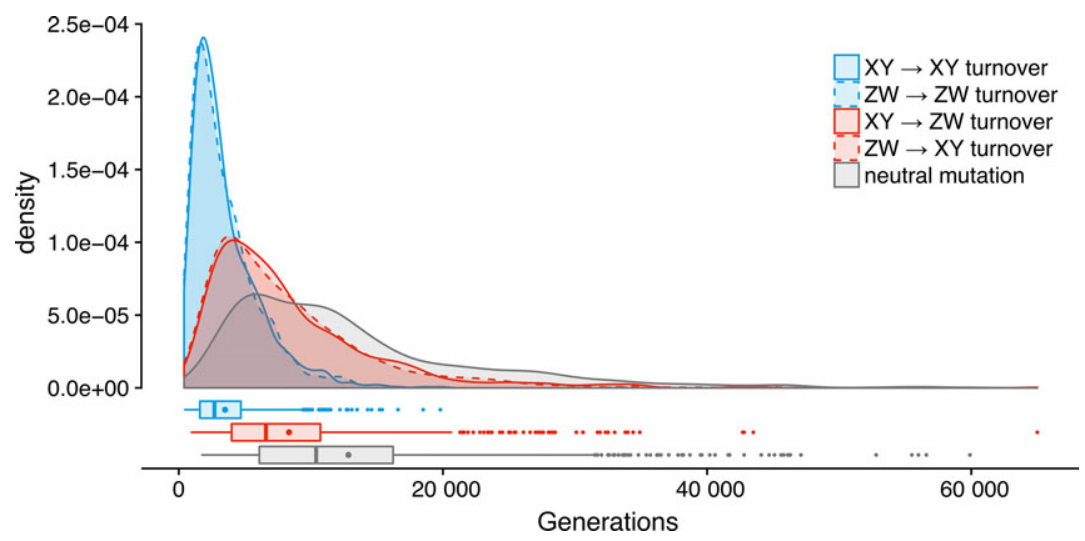

Fig. 1 Distribution of the time to cis-heterogamety turnovers (blue), trans-heterogamety turnovers (red) and fixation of a neutral allele at an autosomal locus (grey); across 1000 replicates with random mating and $N=1000$. On the boxplots are shown the median (thick line) and mean (dot) for XY to XY turnovers (blue), XY to ZW turnovers (red) and neutral mutation (grey).

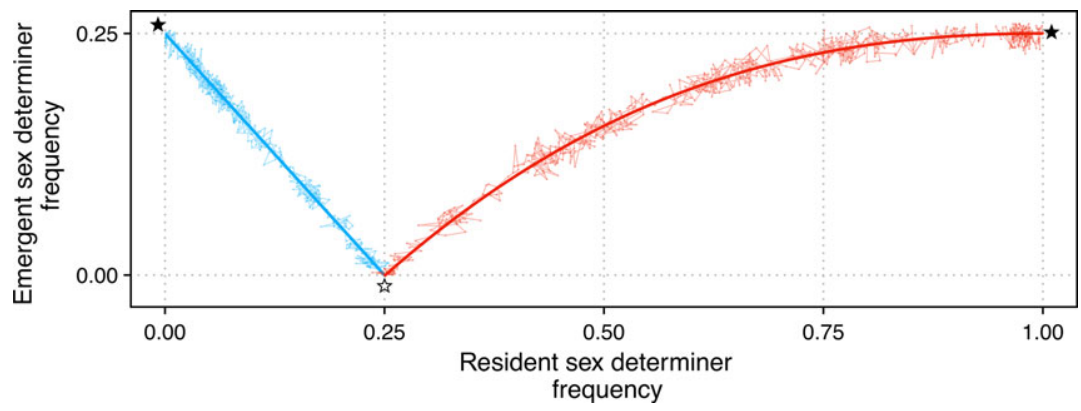

Fig. 2 Neutral models for an XY to XY turnover (left, blue) and an XY to ZW turnover (right, red). The thick lines show the theoretical paths of neutral equilibria, represented as the change in frequency of the emergent sex determiner relative to the ancestral $\mathrm{Y}$ chromosome, throughout a turnover. The equation for the equilibrium path for the XY to ZW turnover was obtained using the equilibrium set of frequencies for genotypes in that kind of turnover, given in Bull \& Charnov (1977), and solving the frequency of the feminizing $F\left(\mathrm{fq}(F)=\frac{\mathrm{XX} \mathrm{aF}}{2}+\frac{X Y \mathrm{aF}}{2}+\frac{\mathrm{YY} \mathrm{aF}}{2}\right)$ as a function of $\mathrm{Y}\left(\mathrm{fq}(Y)=\frac{\mathrm{XYaa}}{2}+\frac{\mathrm{XYaF}}{2}+\mathrm{YYaa}+\mathrm{YYaF}\right): F=\frac{1}{4}\left(-1+2 Y-2 Y^{2}+2 \sqrt{2 Y^{2}-2 Y^{3}+Y^{4}}\right)$. The thin lines show the trajectories taken in a single simulation of each type. The simulations were initiated with a single mutant sex determiner $(\mu=0)$ and run until the turnover had occurred (polymorphism was lost at the ancestral sex determination locus). The unfilled star shows the starting point of simulations, and filled stars the endpoints.

Changing the proportion of breeding males had different impact on all four types of turnovers (Fig. 3B, S5). ZW to ZW turnovers were not affected at all, whereas $\mathrm{XY}$ to $\mathrm{XY}$ turnovers were affected in the same way they were by modifying population size (the 'relative' time to turnover was unaffected, Fig. 3b), in line with the idea the 'drift-induced' selection does not operate here. The two types of transheterogamety turnovers were also affected differently, $\mathrm{XY}$ to ZW turnovers being affected much more strongly than $\mathrm{ZW}$ to $\mathrm{XY}$ turnovers. These results can be understood in the light of the effect that decreasing the proportion of breeding males has on the effective size of the $\mathrm{Y}$ chromosome, and to a lesser extent, autosomes (Nunney, 1993). In trans-heterogamety turnovers, as one of the two sex determiners is malelimited, a decrease in the number of breeding males results in greater fluctuations around the line of neutral equilibria (Fig. S3), causing sex ratio variations to increase (Fig. S4). The decrease in time to XY to ZW turnovers can therefore be explained by increased 'drift-induced' selection. In ZW to XY turnovers, however, this effect is limited as polygyny increases the probability that the emergent male sex determiner is lost when rare (e.g. with $10 / 500$ breeding males, it has a $98 \%$ chance of being lost at the first generation when it appears by mutation).

To summarize, we found that for the vast majority of parameters tested, neutral sex chromosome turnovers triggered by epistatically dominant mutations occur faster than substitutions at neutral loci not involved in sex determination and that the evolutionary dynamics of cis- and trans-heterogamety turnovers differ, the former being more likely to occur than the latter (Fig. 2). 

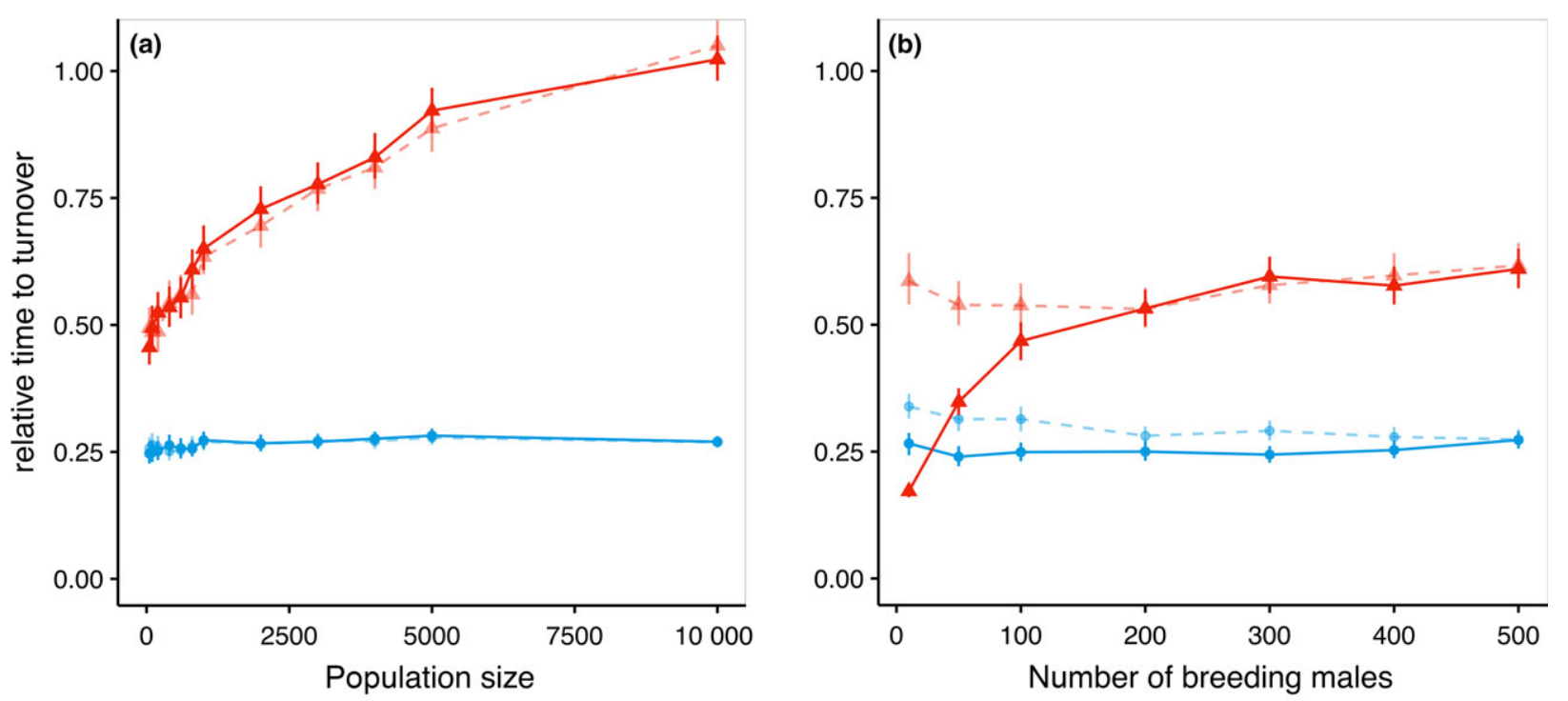

Turnover type

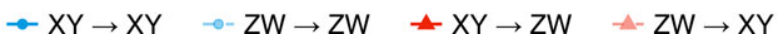

Fig. 3 Mean time to turnover relative to mean time to fixation of a neutral mutation at an autosomal locus (across 1000 replicates each), as a function of (a) population size (here mating is random) and (b) number of breeding males $(N=1000)$. Error bars show bootstrap $95 \%$ confidence interval.

There were, however, some exceptions: trans-heterogamety turnovers could become more difficult to achieve than neutral autosomal substitution in very large populations $(N>10000)$. In contrast, $\mathrm{XY}$ to $\mathrm{ZW}$ turnovers could become relatively more likely than $X Y$ to $\mathrm{XY}$ turnovers in cases of extreme polygyny (10 breeding males in a population of 1000 individuals). Although such strong polygyny must be fairly rare, many other demographic and ecological processes tend to reduce effective population size (such as fluctuations in population size, departures from a $1: 1$ sex ratio, age-structured breeding or population subdivisions; see Caballero, 1994; Storz et al., 2001; Waples, 2016; and references therein), so situations in which trans-heterogamety turnovers are more likely to occur could occasionally arise in nature.

The patterns observed for epistatically dominant mutants differed somewhat in our simulations with mildly or weakly determining mutants. In all four cases analysed, with $N=1000$, the mean time to turnover with these nondominant mutants was greater than for epistatically dominant mutants and closer to (or even greater than) that of neutral autosomal mutations (Fig. S6). The reason for this difference is that the paths of neutral equilibria are longer when the mutant sex determiner is not dominant, because it has to reach a higher frequency for the turnover to be achieved: 0.75 and 1 for sex-determining mutations with mild and weak effects, respectively (Fig. S7). This suggests that, assuming the same rate of emergence, dominant sex-determining mutations are more likely to trigger neutral sex chromosome turnovers than nondominant ones. Furthermore, these four types of turnovers involving nondominant mutations are affected by the drift-induced selective force (as shown by the impact of population size on relative time to turnover; Fig. S6), but the impact of this force varies with the type of mutant. Additional work is required to further expand these preliminary observations.

What general predictions can we make from these results? First, if transitions among sex determination systems are neutral and primarily caused by epistatically dominant mutant sex determiners, then the heterogamety pattern is likely to be conserved across species (assuming a similar rate of emergence of mutant male and female sex determiners). Interestingly, this seems to be the case in several vertebrate lineages that experience frequent turnovers (see Introduction). Furthermore, if XY systems evolve more easily from hermaphroditism than ZW systems (Charlesworth \& Mank, 2010; Bachtrog et al., 2011), then neutral turnovers can also account for the higher prevalence of male heterogamety in animals and plants (Ashman et al., 2014). It is worth recalling, however, that other causes have been invoked to account for the predominance of XY systems. For instance, female heterogamety might simply be more difficult to detect with the current methods based on structural and sequences differences between the sex chromosomes, as W chromosomes are expected to evolve more slowly than 
Y chromosomes (e.g. because of weaker sexual selection and lower germ-line mutation rate in females; Bachtrog et al., 2011; Ellegren, 2011; Mank, 2012).

Second, drift-mediated turnovers should be more frequent in species with small effective population size. An obvious reason is that although selection is less efficient if effective size is small, the effect of drift becomes relatively stronger. Trans-heterogamety turnovers gain an additional selective advantage at low effective population size stemming from selection for a balanced sex ratio (assuming the mutant sex determiner is dominant; Veller et al., 2017). This also implies that in a large population, when a new sex determiner emerges and there are no fitness differences between sexual genotypes, loss of polymorphism at one of the two sex-determining loci will take more time. Therefore, if drift plays a major role in sex chromosome turnovers, polygenic sex determination systems should be more frequent in species with large effective population size. Unfortunately, too few polygenic sex determination systems have been described so far (Moore $\&$ Roberts, 2013) to test this prediction.

As a concluding message, it is worth recalling that determining the relative impact of neutral and selective processes as drivers of evolutionary changes has been at the centre of discussions for a long time in evolutionary biology. Drift-mediated effects probably play only a minor role in sex chromosome turnovers if the resident or emergent sex-determining chromosome has a selective advantage over the other (for instance, the existence of a male beneficial sexually antagonistic allele on a resident $\mathrm{Y}$ chromosome, even if it has a very weak selective disadvantage in females $(s=0.005)$, is enough to completely prevent $\mathrm{XY}$ to $\mathrm{ZW}$ turnovers in models similar to those presented in this paper, with $N=1000$, unpublished data). Empirically characterizing the evolutionary cause(s) of a sex chromosome turnover based on single turnover events is difficult, because once a transition is over, the presence (or absence) of a fitness advantage associated with the emergent sex determiner cannot be evaluated. A better understanding of the forces driving turnovers might come from analysing patterns of transitions types in clades that experience frequent turnovers, at a macro-evolutionary scale. With this in mind, we strongly encourage researchers that use genotyping-by-sequencing methods to identify sex determination systems to also search for the chromosomal pair carrying the sex determiner(s) (e.g. Brelsford et al., 2017). This should help having a more precise idea of the relative frequency of cis- and trans-heterogamety turnovers and might give us a chance of assess the relative role of neutral and non-neutral forces in sex chromosome turnovers.

\section{Acknowledgments}

We are thankful to J. Goudet and C. Mullon for stimulating discussions and helpful comments. The computations were performed at the Vital-IT (http://www.vital-it.ch) Center for high-performance computing of the SIB Swiss Institute of Bioinformatics. Funding was provided by the Swiss National Science Foundation (Grant Number 31003A_166323).

\section{References}

Bachtrog, D., Kirkpatrick, M., Mank, J.E., McDaniel, S.F., Pires, J.C., Rice, W. et al. 2011. Are all sex chromosomes created equal? Trends Genet. 27: 350-357.

Bachtrog, D., Mank, J.E., Peichel, C.L., Kirkpatrick, M., Otto, S.P., Ashman, T.-L. et al. 2014. Sex determination: why so many ways of doing it? PLoS Biol. 12: e1001899.

Becking, T., Giraud, I., Raimond, M., Moumen, B., Chandler, C., Cordaux, R. et al. 2017. Diversity and evolution of sex determination systems in terrestrial isopods. Sci. Rep. 7: 1084 .

Beukeboom, L.W. \& Perrin, N. 2014. The Evolution of Sex Determination. Oxford University Press, Oxford.

Blaser, O., Grossen, C., Neuenschwander, S. \& Perrin, N. 2012. Sex-chromosome turnovers induced by deleterious mutation load. Evolution 67: 635-645.

Brelsford, A., Lavanchy, G., Sermier, R., Rausch, A. \& Perrin, N. 2017. Identifying homomorphic sex chromosomes from wild-caught adults with limited genomic resources. Mol. Ecol. Resour. 17: 752-759.

Bull, J.J. \& Charnov, E.L. 1977. Changes in the heterogametic mechanism of sex determination. Heredity 39: 1-14.

Caballero, A. 1994. Developments in the prediction of effective population size. Heredity 73: 657-679.

Charlesworth, D. \& Mank, J.E. 2010. The birds and the bees and the flowers and the trees: lessons from genetic mapping of sex determination in plants and animals. Genetics $\mathbf{1 8 6}$ 9-31.

Cordaux, R., Bouchon, D. \& Grève, P. 2011. The impact of endosymbionts on the evolution of host sex-determination mechanisms. Trends Genet. 27: 332-341.

van Doorn, G.S. 2014a. Patterns and mechanisms of evolutionary transitions between genetic sex-determining systems. Cold Spring Harb. Perspect. Biol. 6: pii: a017681.

van Doorn, G.S. 2014b. Evolutionary transitions between sexdetermining mechanisms: a review of theory. Sex. Dev. 8: 7-19.

van Doorn, G.S. \& Kirkpatrick, M. 2007. Turnover of sex chromosomes induced by sexual conflict. Nature 449: 909-912.

van Doorn, G.S. \& Kirkpatrick, M. 2010. Transitions between male and female heterogamety caused by sex-antagonistic selection. Genetics 186: 629-645.

Dufresnes, C., Bertholet, Y., Wassef, J., Ghali, K., Savary, R., Pasteur, B. et al. 2014. Sex-chromosome differentiation parallels postglacial range expansion in European tree frogs (Hyla arborea). Evolution 68: 3445-3456.

Ellegren, H. 2011. Sex-chromosome evolution: recent progress and the influence of male and female heterogamety. Nat Rev. Genet. 12: 157-166.

Ewens, W.J. 2012. Mathematical Population Genetics 1: Theoretical Introduction. Springer, New York.

Ezaz, T., Sarre, S.D., O'Meally, D., Marshall Graves, J.A. \& Georges, A. 2009. Sex chromosome evolution in lizards: independent origins and rapid transitions. Cytogenet. Genome Res. 127: 249-260. 
Furman, B.L.S. \& Evans, B.J. 2016. Sequential turnovers of sex chromosomes in African Clawed Frogs (Xenopus) suggest some genomic regions are good at sex determination. G3 6: $3625-3633$.

Gamble, T., Coryell, J., Ezaz, T., Lynch, J., Scantlebury, D.P. \& Zarkower, D. 2015. Restriction site-associated DNA sequencing (RAD-seq) reveals an extraordinary number of transitions among gecko sex-determining systems. Mol. Biol. Evol. 32: 1296-1309.

Kozielska, M., Weissing, F.J., Beukeboom, L.W. \& Pen, I. 2010. Segregation distortion and the evolution of sex-determining mechanisms. Heredity 104: 100-112.

Lambert, M.R., Skelly, D.K. \& Ezaz, T. 2016. Sex-linked markers in the North American green frog (Rana clamitans) developed using DArTseq provide early insight into sex chromosome evolution. BMC Genom. 17: 844.

Mank, J.E. 2012. Small but mighty: the evolutionary dynamics of $\mathrm{W}$ and $\mathrm{Y}$ sex chromosomes. Chromosom. Res. 20: 21-33.

Mank, J.E. \& Avise, J.C. 2009. Evolutionary diversity and turn-over of sex determination in teleost fishes. Sex. Dev. 3: $60-67$.

Miura, I. 2008. An evolutionary witness: the frog rana rugosa underwent change of heterogametic sex from $\mathrm{XY}$ male to ZW female. Sex. Dev. 1: 323-331.

Moore, E.C. \& Roberts, R.B. 2013. Polygenic sex determination. Curr. Biol. 23: R510-R512.

Muyle, A., Shearn, R. \& Marais, G.A.B. 2018. The evolution of sex chromosomes and dosage compensation in plants. Genome Biol. Evol. 9: 627-645.

Neuenschwander, S., Hospital, F., Guillaume, F. \& Goudet, J. 2008. quantiNemo: an individual-based program to simulate quantitative traits with explicit genetic architecture in a dynamic metapopulation. Bioinformatics 24: 1552-1553.

Nunney, L. 1993. The influence of mating systems and overlapping generations on effective population size. Evolution 47: 1329-1341.

Phillips, R.B. 2013. Evolution of the sex chromosomes in salmonid fishes. Cytogenet. Genome Res. 141: 177-185.

Pokorná, M. \& Kratochvíl, L. 2009. Phylogeny of sex-determining mechanisms in squamate reptiles: are sex chromosomes an evolutionary trap? Zool. J. Linn. Soc.-Lond. 156: $168-183$.

Roberts, R.B., Ser, J.R. \& Kocher, T.D. 2009. Sexual conflict resolved by invasion of a novel sex determiner in Lake Malawi cichlid fishes. Science 326: 998-1001.

Storz, J.F., Ramakrishnan, U. \& Alberts, S.C. 2001. Determinants of effective population size for loci with different modes of inheritance. J. Hered. 92: 497-502.
Sumida, M. \& Nishioka, M. 2000. Sex-linked genes and linkage maps in amphibians. Comp. Biochem. Physiol. $-B$ Biochem. Mol. Biol. 126: 257-270.

Tree of Sex Consortium, Ashman, T.-L., Bachtrog, D., Blackmon, H., Goldberg, E.E., Hahn, M.W., et al. 2014. Tree of sex: a database of sexual systems. Sci. Data 1: 1-8.

Veller, C., Muralidhar, P., Constable, G.W.A. \& Nowak, M.A. 2017. Drift-induced selection between male and female heterogamety. Genetics 207: 711-727.

Vicoso, B. \& Bachtrog, D. 2015. Numerous transitions of sex chromosomes in Diptera. PLoS Biol. 13: e1002078.

Vuilleumier, S., Lande, R., Van Alphen, J.J.M. \& Seehausen, O. 2007. Invasion and fixation of sex-reversal genes. J. Evol. Biol. 20: 913-920.

Waples, R.S. 2016. Tiny estimates of the Ne/N ratio in marine fishes: are they real? J. Fish Biol. 89: 2479-2504.

\section{Supporting information}

Additional supporting information may be found online in the Supporting Information section at the end of the article.

Figure S1 Expected vs. observed time to fixation of a neutral mutant at an autosomal locus.

Figure S2 Influence of population size on (a) absolute time to turnover, and (b) absolute time to fixation of a neutral autosomal allele.

Figure S3 Frequency of the novel sex determiner as a function of the frequency of the resident sex determiner, throughout different types of turnovers.

Figure S4 Population sex ratio throughout different types of turnovers.

Figure S5 Influence of the proportion of breeding males on (a) absolute time to turnover and (b) absolute time to substitution at neutral autosomal locus.

Figure S6 Influence of dominance relationship among the sex-determining factors on time to turnover.

Figure S7 Paths of neutral equilibria for turnovers triggered by non-dominant sex-determining factors.

Data deposited at Dryad: https://doi.org/10.5061/dryad.62sm982

Received 19 February 2018; revised 23 May 2018; accepted 17 June 2018 